\title{
INCOMPRESSIBLE SURFACES AND THE TOPOLOGY OF 3-DIMENSIONAL MANIFOLDS
}

\author{
IAIN R. AITCHISON and J. HYAM RUBINSTEIN
}

(Received February 23 1993)

\begin{abstract}
Existence and properties of incompressible surfaces in 3-dimensional manifolds are surveyed. Some conjectures of Waldhausen and Thurston concerning such surfaces are stated. An outline is given of the proof that such surfaces can be pulled back by non-zero degree maps between 3-manifolds. The effect of surgery on immersed, incompressible surfaces and on hierarchies is discussed. A characterisation is given of the immersed, incompressible surfaces previously studied by Hass and Scott, which arise naturally with cubings of non-positive curvature.
\end{abstract}

1991 Mathematics subject classification (Amer. Math. Soc.): 57 N 10, 57 M 35, 57 M 50.

\section{Introduction}

We give a brief, eclectic survey of the role of incompressible surfaces in the study of 3-dimensional manifolds.

The solution of Dehn's Lemma and its extension to the Loop Theorem by Papakyriakopoulos in $1957,[34,35]$, ushered in a period of intense activity. Incompressible embedded surfaces and hierarchies were introduced by Haken $[12,14]$ (see also Schubert [37]) and used by Waldhausen [48] to show that members of the class of Haken 3-manifolds are completely determined by their fundamental group. Jaco and Shalen [23] and Johannson [24] independently gave the characteristic variety decomposition of Haken 3-manifolds, as suggested by Waldhausen [50]. This is obtained by splitting such 3-manifolds along

(C) 1993 Australian Mathematical Society 0263-6115/93 \$A2.00+0.00 
embedded incompressible annuli and tori. Finally Thurston [45, 46, 47] used the hierarchy of incompressible surfaces in simple Haken 3-manifolds to obtain his celebrated uniformisation theorem: such 3-manifolds have hyperbolic metrics. More generally he described special Riemannian metrics based on his eight geometries, which occur for basic classes of 3-manifolds (see Scott [39] for an excellent survey of Thurston's geometrisation program).

To progress beyond the class of Haken manifolds, Waldhausen and Thurston have suggested that immersed incompressible surfaces should play a pivotal role. In this paper, we begin in Section 1 with a very rapid review of the basic theory of embedded incompressible surfaces. In the second section, special classes and properties of immersed incompressible surfaces are discussed, including well-known conjectures of Waldhausen and Thurston. In the last section recent results are described, including unpublished work about immersed incompressible surfaces arising from the polyhedral metrics of non-positive curvature studied by the authors in $[1,2,3,4,5]$. The two key theorems are a combinatorial version of the Loop Theorem applying to immersed surfaces and a necessary and sufficient condition for the existence of an immersed, incompressible surface in a 3-dimensional manifold. Applications are given to the survival of immersed, incompressible surfaces and hierarchies under surgery, and also under pull-back by non-zero degree maps between 3-manifolds.

We would like to thank G.A. Swarup for helpful comments.

\section{Embedded incompressible surfaces}

A classical result of Moise [31] is that every 3-dimensional manifold has a triangulation which is unique up to subdivision. So we will view each 3manifold $M$ as a simplicial complex, that is, a union of tetrahedra (3-simplices) which possibly intersect in vertices, edges or faces. A 3-simplex is oriented by a choice of ordering of its vertices, up to any even permutation. This induces an orientation on all of the faces of the 3-simplex. A 3-manifold $M$ is orientable if all of its 3 -simplices can be oriented so that the induced orientations on any face coming from the pair of 3-simplices containing that face are opposite. $M$ is compact if and only if it is a finite simplicial complex. The boundary of $M$, denoted $\partial M$, is the collection of faces not belonging to a pair of simplices. $M$ is closed if it is compact and has no boundary. From now on we will assume for simplicity that all our 3-manifolds are orientable. Also all maps between 3-manifolds will be supposed to be piecewise-linear 
(PL): for appropriate subdivision of domain and range, maps will be simplicial, taking vertices to vertices and simplices to simplices in an affine linear way. Similarly curves or surfaces embedded in 3-manifolds will be PL subcomplexes in appropriate subdivisions. Throughout, $S^{n}$ denotes the $n$-sphere.

2.1. Reduction to the irreducible case. If $M_{1}$ and $M_{2}$ are 3-manifolds, their connected sum, denoted $M_{1} \# M_{2}$, is obtained by removing small, open 3-cells from each, and gluing the resulting manifolds along their boundary 2-spheres. Conversely, if an embedded 2-sphere $S$ separates $M$ into pieces $M_{1}^{\prime}$ and $M_{2}^{\prime}$, then we can add 3-cells to $M_{1}^{\prime}$ and $M_{2}^{\prime}$ to form $M_{1}$ and $M_{2}$ so that $M$ is homeomorphic to $M_{1} \# M_{2} . S$ is called an essential 2-sphere (sometimes an incompressible 2sphere) if either $S$ separates $M$ and neither $M_{1}^{\prime}$ nor $M_{2}^{\prime}$ is a 3-cell, or if $S$ does not separate $M . M$ is called irreducible if it has no essential 2 -spheres, and is called prime if it has no essential separating 2-spheres. The 3-sphere $S^{3}$ has the property that $M \# S^{3}$ is homeomorphic to $M$.

In 1928, Kneser [25] showed that any closed 3-manifold $M$ can be written as a finite connected sum $M_{1} \# \ldots \# M_{k}$, where each $M_{i}$ is either irreducible and not $S^{3}$ or a copy of $S^{1} \times S^{2}$, or a twisted product $S^{1} \tilde{x} S^{2}$. In 1957, Milnor [30] proved that Kneser's decomposition is unique: the summands of two such connected sum decompositions for $M$ correspond, after reordering if necessary.

Hence to classify 3-manifolds, it suffices to study the irreducible case, which we will do from now on.

2.2. The fundamental group. The fundamental classification conjecture about 3-manifolds is the following:

CONJECTURE 2.1. If $M, M^{\prime}$ are closed irreducible 3-manifolds, and $\pi_{1}(M)$, $\pi_{1}\left(M^{\prime}\right)$ are isomorphic infinite groups, then $M$ is homeomorphic to $M^{\prime}$.

REMARK 2.1. (1) By the Sphere Theorem of Papakyriakopoulos [34], existence of a non-trivial element of $\pi_{2}(M)$ implies that some non-trivial element can be represented by an embedded 2-sphere. It is then not difficult to show that the 3-manifolds of the conjecture are aspherical (Eilenberg-MacLane spaces), that is, all higher homotopy groups vanish, using Whitehead's Theorem. Two such 3-manifolds are thus homotopy equivalent if and only if their fundamental groups are isomorphic. This conjecture then states that homotopy equivalence implies homeomorphism.

(2) The set of fundamental groups arising from all closed $n$-manifolds for $n \geq 4$ is the set of all finitely presented groups. Since Markov has shown that 
the isomorphy problem for two such groups is undecidable [28] , the problem of deciding if two closed $n$-manifolds are homeomorphic is undecidable. On the other hand, the restricted nature of 3-manifold fundamental groups makes it plausible that the homeomorphism problem in dimension 3 is decidable. Haken [14] gave a positive solution for the class of Haken 3-manifolds, using work of Hemion [17]. Note that if Thurston's Geometrisation Program $[45,46]$ is correct then the homeomorphism problem should be solvable. Note that all 3-manifold fundamental groups should be residually finite - see Hempel [20].

2.3. Incompressible surfaces, Dehn's Lemma and the Loop Theorem. We will give the most commonly used consequence of the combined version of Dehn's Lemma and the Loop Theorem, due to Stallings [42]. An incorrect proof of Dehn's Lemma was given by Dehn in 1910 and a full proof only found by Papakyriakopoulos in 1956. (See Hempel's book [18] for a proof).

A compact surface $L$ with non-empty boundary is properly embedded in $M$ if $L \cap \partial M=\partial L$. Let $i: L \rightarrow M$ denote the embedding of $L$ in $M$.

THEOREM 2.1. Assume $L$ is a compact orientable surface which

(1) is not a disk or 2-sphere and

(2) is either closed or properly embedded in a compact orientable 3manifold $M$.

Then the homomorphism $i_{*}: \pi_{1}(L) \rightarrow \pi_{1}(M)$ is one-to-one if and only if any disk $D$ embedded in $M$ with $D \cap L=\partial D$ has boundary $\partial D$ contractible in $L$.

REMARK 2.2. A surface $L$ as in the theorem is called incompressible. This result can be paraphrased as saying that algebraic incompressibility is equivalent to geometric incompressibility.

An important result proved using the same approach is

THEOREM 2.2. Suppose $L$ is a compact orientable surface with non-empty boundary, other than a disk, properly embedded in $M$. Then $i_{*}: \pi_{1}(L, \partial L) \rightarrow$ $\pi_{1}(M, \partial M)$ is one to one if and only if for any disk $D$ embedded in $M$ with $\partial D=a \cup b$, for arcs $a, b$, where $D \cap L=a$ and $D \cap \partial M=b$, there is an arc $g$ in $\partial L$ with the same endpoints $a s a, b$ and with $a, b$ homotopic, keeping ends fixed, to $g$ in $L, \partial M$ respectively.

A surface $L$ satisfying the conditions of Theorem 2.2 is said to be boundary incompressible. Theorem 2.2 shows that algebraic and geometric versions of 
this concept are equivalent. Note that $\pi_{1}(L, \partial L)$ is the set of equivalence classes of arcs in $L$ with ends on $\partial L$, under the relation of homotopy.

For closed, orientable, irreducible 3-manifolds, Waldhausen [48] gave an important criterion for the existence of closed, embedded, orientable, incompressible surfaces.

THEOREM 2.3. Suppose $M$ is a closed, orientable, irreducible 3-manifold. Then $M$ has a closed, orientable, embedded, incompressible surface $L$, if and only if $\pi_{1}(M)$ surjects homomorphically onto either

(1) an amalgamated free product $A *_{C} B$ or

(2) an $H N N$ extension $A *_{C}$,

where $C$ is isomorphic to $\pi_{1}(L)$. The first case occurs when $L$ separates $M$, and the second when $L$ is non-separating.

REMARK 2.3. Extensions of this result are known in case $L$ or $M$ (or both) have non-empty boundary.

REMARK 2.4. By a technique of Stallings [43], the condition that $\pi_{1}(M)$ surjects onto such an HNN extension is equivalent to $H_{1}(M, Z)$ being infinite. The latter condition is particularly easy to verify in specific examples. An important approach to finding separating incompressible surfaces was given by Culler and Shalen [6].

2.4. Hierarchies. A Haken 3-manifold is a compact, orientable, irreducible 3-manifold which either

(1) has non-empty boundary which is a collection of incompressible surfaces or

(2) is closed, and admits an embedded, closed, orientable, incompressible surface.

EXAMPLE 2.1. An important class of Haken 3-manifolds is given by knot and link complements. We work with the 3 -sphere $S^{3}$, viewed as the one-point compactification of Euclidean 3-space $R^{3}$. A knot (respectively link) is an embedded circle (respectively, a finite collection of disjoint, embedded circles) in $S^{3}$. If the link is not splittable (that is, there is no 2-sphere embedded in $S^{3}$ disjoint from the link and separating the components), then the complement is irreducible. If a small, open, tubular neighbourhood of the knot or link is removed from $S^{3}$, we obtain the complement, with boundary a collection of tori. If no component bounds an embedded disk in the complement of the other 
components, then the boundary tori of the complement are incompressible. So all such knot and link complements are Haken 3-manifolds. By a powerful result of Gordon and Luecke [10], if two knot complements are homeomorphic, then the corresponding knots are equivalent, in the sense that there is a homeomorphism of $S^{3}$ to itself taking one knot to the other. So the classification of knots reduces to that of their complements. On the other hand, links of more than one component admit no such classification.

Recall that in an elementary study of surfaces, an important technique is to cut open a closed surface along an embedded essential loop (that is, a noncontractible curve), and then to further cut open along essential arcs, to obtain a disc. The disc so obtained carries additional information recording the sequence of cuts, which give it the structure of a polygon. Important invariants of the surface, such as the Euler characteristic and genus, can be deduced from the number of vertices and arcs arising in this procedure.

For Haken 3-manifolds there is a similar procedure, based on a hierarchy. A hierarchy is a collection of embedded incompressible surfaces. Suppose $M$ is a Haken 3-manifold. If $M$ is closed, choose a collection of disjoint, closed, orientable, incompressible surfaces . Now cut $M$ open along these surfaces by removing a small open product neighbourhood to obtain $M_{1}$. If $\partial M \neq \emptyset$, set $M=M_{1}$. In both cases, $M_{1}$ is Haken with non-empty boundary. Choose disjoint, properly embedded, compact, orientable, incompressible surfaces in $M_{1}$ and cut open along these surfaces to obtain $M_{2}$. By results of Haken [13] and Kneser [25], this procedure can only be applied finitely many times before obtaining some $M_{k}$, which is a union of a finite number of 3-dimensional polyhedra homeomorphic to 3-cells. The total collection of these incompressible surfaces is called a hierarchy for $M$. It is shown in Jaco's book [21] that one can always choose $k \leq 4$.

Waldhausen [48] used an inductive procedure based on hierarchies to show:

THEOREM 2.4. If $M, M^{\prime}$ are compact, orientable, irreducible 3-manifolds, with $M$ Haken, and there is a relative homotopy equivalence $(M, \partial M)$ to $\left(M^{\prime}, \partial M^{\prime}\right)$, then $M$ and $M^{\prime}$ are homeomorphic.

REMARK 2.5. This establishes Conjecture 2.1 in the case where $M$ and $M^{\prime}$ are closed, and $M$ is Haken.

2.5. Characteristic varieties and geometrisation. We give a very brief, rough discussion for completeness. 
A Seifert fibering of a 3-manifold $M$ is a foliation of $M$ by circles (see Epstein [7], Seifert [40], Orlik [33]). Seifert [40] gave a complete classification of 3-manifolds with such fiberings, which are now called Seifert fibre spaces.

Given a Haken 3-manifold $M$, its characteristic variety $V$ is a maximal embedded 3-submanifold which is

(1) a Seifert fibre space, such that

(2) $V \cap \partial M$ consists of fibred tori and annuli in $\partial V$, and

(3) the closure of $\partial V-\partial M$ consists of properly embedded incompressible annuli and tori.

It was proved independently by Jaco and Shalen [23], and Johannson [24], that $V$ is uniquely determined in $M$. If $V$ is empty then $M$ is called a simple Haken 3-manifold.

Recall that a Riemannian metric with constant negative sectional curvature -1 is called a hyperbolic metric.

One version of Thurston's Uniformisation Theorem is as follows:

THEOREM 2.5. The interior of any simple, Haken 3-manifold $M$ with only toral boundary components (if any) admits a complete hyperbolic metric of finite volume.

REMARK 2.6. Prior to Thurston's work, Johannson had extended Waldhausen's result (Theorem 2.4 above) to simple Haken 3-manifolds, using a homotopy equivalence from $M$ to $M^{\prime}$ instead of a (stronger) relative homotopy equivalence from $(M, \partial M)$ to $\left(M^{\prime}, \partial M^{\prime}\right)$. Note that Johannson's Theorem follows from Thurston's Theorem (Theorem 2.5) together with Mostow rigidity [32], which states that finite-volume hyperbolic manifolds with isomorphic fundamental groups are isometric. Their results apply to the important class of simple knot complements. A simple proof of Johannson's Theorem can be found in [44].

\section{Immersed incompressible surfaces}

Thurston [45] has given the following heuristic argument that in some sense, 'most' 3-manifolds do not admit embedded incompressible surfaces. A classical result of Wallace and Lickorish [26], states that every closed orientable 3-manifold can be obtained by Dehn surgery on a link in $S^{3}$. Such surgery is performed by removing disjoint, open, solid tori neighbourhoods of the components of the link and gluing the solid tori back in by self-homeomorphisms of the 
boundary tori. Hatcher [16] showed that most such surgeries yield 3-manifolds for which any closed, embedded, incompressible surface must lie in the link complement. So if there are no such surfaces disjoint from the link, then the resulting 3-manifold cannot be Haken.

A fundamental conjecture, first made by Waldhausen [49], and extensively discussed by Thurston, is

CONJECTURE 3.1. If $M$ is a closed, orientable, irreducible 3-manifold with infinite fundamental group, then $M$ has a finite-sheeted cover $\tilde{M}$ which is Haken.

By projecting an embedded, incompressible surface in $\tilde{M}$ we obtain an immersed, incompressible surface in $M$. So a weaker version of this conjecture is:

CONJECTURE 3.2. Under the same assumptions on $M$, there is an immersed closed orientable incompressible surface in $M$.

On the other hand, the conditions given in Theorem 4.3 for the existence of embedded incompressible surfaces are difficult to check in general. An easier approach is suggested by a stronger conjecture.

CONJECTURE 3.3. Under the same assumptions on $M$, there is a finite-sheeted cover $\tilde{M}$ with $H_{1}(\tilde{M}, Z)$ infinite.

This is equivalent to $\tilde{M}$ having a non-separating, closed, embedded, incompressible surface. The simplest case in which this occurs is when $\tilde{M}$ is in fact a fibre bundle over the circle. This leads to an even stronger conjecture made by Thurston.

CONJECTURE 3.4. With the same hypotheses on $M$, there is a finite-sheeted cover $\tilde{M}$ which is a closed, orientable, surface bundle over the circle.

We will briefly review some recent results on these conjectures and look at some implications of Conjecture 3.1 being true.

Conjecture 3.3 is true in the special case that $M$ has a finite-sheeted cover $\tilde{M}$ which admits an orientation-reversing involution (see Millson [29] and Hempel [19]).

The authors [3] have shown that Conjecture 3.2 is true for all closed, orientable 3-manifolds which admit polyhedral metrics of non-positive curvature arising from cubings. 
To discuss other results, we review some concepts from hyperbolic geometry. An orientable hyperbolic 3-manifold $M$ has universal covering space hyperbolic 3-space $H^{3}$, with $\pi_{1}(M)$ acting by isometries as the covering transformation group. Thus $\pi_{1}(M)$ is isomorphic to a discrete torsion-free subgroup of $\operatorname{PSL}(2, C)$, the isometry group of $H^{3}$. An immersed, orientable, closed, incompressible surface $f: L \rightarrow M$ determines a subgroup $f_{*}\left(\pi_{1}(L)\right)$ of $\pi_{1}(M)$, and hence of $P S L(2, C)$. If this subgroup is conjugate into $\operatorname{PS} L(2, R)$, then $L$ is a totally geodesic (or Fuchsian) surface in $M$. The universal covering $\tilde{L}$ of $L$ lifts to the universal cover $H^{3}$ of $M$ and the image of the map $\tilde{f}: \tilde{L} \rightarrow H^{3}$ has a limit set in the sphere at infinity. If this limit set is a quasi-circle, then $L$ is a quasi-Fuchsian or geometrically finite surface. Otherwise, the limit set is the whole sphere, and $L$ is said to be geometrically infinite. The complement of the closure of the limit set is the domain of discontinuity. An important result of Thurston [45] is that if $M$ contains an incompressible, geometrically infinite surface, then $M$ is finitely covered by a surface bundle over a circle, with this surface corresponding to the fibre. Hence Conjecture 3.4 is true. As the fibre of a hyperbolic surface bundle is always of this type, Conjecture 3.4 is equivalent in the hyperbolic case to the conjecture that a hyperbolic 3-manifold contains a geometrically infinite incompressible surface. In fact, each of the above conjectures can be made in the special case that $M$ is hyperbolic.

A well-known consequence of Conjecture 3.1 is that if $M$ is non-Haken, and is finitely covered by a Haken 3-manifold, then either $M$ is a Seifert fibred space or $M$ is homotopy equivalent to a hyperbolic 3-manifold (see for example Culler and Shalen [6]). Recently Gabai [9] has shown that if $M$ is homotopy equivalent to a hyperbolic 3-manifold $M^{\prime}$, then $M$ and $M^{\prime}$ have homeomorphic finite-sheeted covers.

Long [27] has shown that if $M$ is a closed, orientable hyperbolic 3-manifold containing an immersed, totally geodesic surface, then $M$ has a finite-sheeted cover in which the lifted surface is embedded. The cover is thus a Haken 3manifold and Conjecture 3.1 follows in this case, since totally geodesic surfaces are automatically incompressible.

Aitchison, Lumsden and Rubinstein [1] showed that a large class of alternating link complements in $S^{3}$ contain immersed closed surfaces, totally geodesic with respect to a natural polyhedral metric. A finite explicit list of excluded surgery coefficients can be given for each component, determined by the combinatorics of a link projection, such that the immersed surface continues to be $\pi_{1}$-injective in the surgered manifolds.

This construction can be generalized. In the case of a hyperbolic link comple- 
ment, there is the following unpublished theorem of Thurston. Suppose $M$ is a hyperbolic 3-manifold which is the interior of a compact, orientable 3-manifold $M^{*}$ with $\partial M^{*}$ consisting of incompressible tori. Assume $f: L \rightarrow M$ is an immersion of a closed, orientable, incompressible surface. $L$ is said to contain an accidental parabolic if there is a an essential loop on $L$ homotopic into $\partial M^{*}$.

THEOREM 3.1. If $f: L \rightarrow M$ has no accidental parabolics, then for all but finitely many surgeries on any tori in $\partial M^{*}, L$ remains incompressible in the surgered manifold.

An abstract bound can be given on the number of surgeries excluded for each component, but an explicit list of surgeries requires more information on cusp geometry.

Another version of Conjecture 3.1 for hyperbolic 3-manifolds, again due to Thurston, has not been so widely publicised. There is an intriguing connection with the results on cubings in [3]. A very easy unpublished result of Thurston is:

THEOREM 3.2. Suppose a hyperbolic 3-manifold $M$ contains an immersed surface $f: L \rightarrow M$ such that all principal curvatures of $L$ have absolute value less than one. Then $L$ is incompressible.

REMARK 3.1. For a totally geodesic surface, all such curvatures are zero. It is easy to show that a surface $L$ as in Theorem 3.2 must be quasi-Fuchsian. Note the pre-image of $L$ in $H^{3}$ cannot have any saddle tangencies relative to a horospherical foliation, so $L$ must be incompressible.

CONJECTURE 3.5. Assume $M$ is a complete, hyperbolic 3-manifold with finite volume. Then $M$ has an immersed, closed, orientable surface with all principal curvatures having absolute value less than one.

REMARK 3.2. With only a little evidence, we conjecture that for hyperbolic 3-manifolds which admit cubings of non-positive curvature, the immersed, incompressible surfaces described in [3] have principal curvatures with absolute value less than one. These surfaces are known to be quasi-Fuchsian.

In the next section we describe a strong 'combinatorial' version of this theorem, useful for constructing many examples of immersed, incompressible surfaces in closed 3-manifolds obtained by surgery. 
3.1. Topological rigidity and least area surface methods. To complete this section, we briefly discuss properties of immersed, incompressible surfaces and applications to Conjecture 2.1 (topological rigidity). We always assume, without loss of generality, that immersions are in general position.

In [8], Freedman, Hass and Scott used minimal surface theory to analyse immersed, incompressible surfaces. Suppose $M$ is endowed with a Riemannian metric. Let $f: L \rightarrow M$ be an immersed, incompressible surface, with $g$ homotopic to $f$ chosen to minimize the area of $g(L)$, among all maps in the homotopy class of $f$. In [8] it is proved that $g$ then has the smallest number of singularities (in a suitable sense) amongst maps homotopic to $f$. Let $M_{L}$ denote the covering of $M$ corresponding to the subgroup $f_{*} \pi_{1}(L)$ of $\pi_{1}(M)$. Another important result is that $g$ lifts to an embedding $g: L \rightarrow M_{L}$. Consequently $g$ lifts to an embedding $g_{L}: \tilde{L} \rightarrow \tilde{M}$, where $\tilde{L}, \tilde{M}$ are the universal coverings of $L, M$. Therefore the pre-image of $g(L)$ in $\tilde{M}$ is a union of embedded planes, set-wise preserved by the action of $\pi_{1}(M)$ as covering transformations.

Note that all the results in [8] can be obtained by using the theory of PL minimal surfaces developed in [22].

In [15], Hass and Scott studied some special classes of immersed, incompressible surfaces, analysing the configurations of embedded planes in the universal covering space.

DEFINITION 3.1. An immersed, closed, orientable, incompressible surface $f: L \rightarrow M$ in a closed, orientable, irreducible 3-manifold satisfies the $n$-plane property if the pre-image of $f(L)$ in the universal covering $\tilde{M}$ consists of embedded planes, and any collection of $n$ of these planes contains a disjoint pair.

$f: L \rightarrow M$ satisfies the 1-line property if the pre-image of $f(L)$ in $\tilde{M}$ consists of embedded planes, any pair of which meet in a single line, or are disjoint.

The following fundamental result of Hass and Scott, substantially generalizing Waldhausen's result in [48], answers Conjecture 1 in case $M$ possesses a certain type of immersed, incompressible surface.

THEOREM 3.3. Suppose $M, M^{\prime}$ are closed, orientable, irreducible 3-manifolds which are homotopy equivalent, with $M$ containing an immersed, closed, orientable, incompressible surface, satisfying the 4-plane and 1-line properties. Then $M$ is homeomorphic to $M^{\prime}$. 
REMARK 3.3. In [3], it is observed that any 3-manifold with a cubing of nonpositive curvature contains such an incompressible surface. This gives a large class of examples to which this theorem applies. (See also the next section.)

Finally we introduce the triple point condition for immersed incompressible surfaces. An incorrect formulation of this was given in [1] and we thank P. Scott for pointing this out.

DEFINITION 3.2. Suppose $f: L \rightarrow M$ is an immersion of a closed, orientable, incompressible surface in a closed, orientable, irreducible 3-manifold, with $f(L)$ having as pre-image a collection of embedded planes in $\tilde{M}$, satisfying the 1 -line property. Then $f$ has the triple point property if whenever three of the planes meet pairwise, the three planes together intersect in an odd number of triple points.

In [3] it is shown that $M$ has a cubing of non-positive curvature if and only if $M$ admits an immersed, incompressible surface $L$ satisfying the 4-plane, 1-line and triple point properties, such that $M-L$ is a union of open 3-cells.

\section{Characterising hierarchies and immersed incompressible surfaces}

The local picture of how the surfaces in a hierarchy fit together is given in Figure 1.

When a 3-manifold is cut open along the surfaces of the hierarchy, a graph is seen on the boundary which is called the boundary pattern (Figure 1). In Waldhausen's solution of the word problem in the fundamental group of Haken 3-manifolds [49], he made an important restriction on the choice of hierarchy. Waldhausen observes that it can be arranged so that there are no triangular regions in the boundary pattern, and that if $D$ is a disk embedded in $M$, intersecting the hierarchy exactly in $\partial D$, and with $\partial D$ crossing the boundary pattern at most three times, then $D$ is parallel into the hierarchy (see Figure 2).

We call a hierarchy nice if it satisfies these two conditions of Waldhausen. Nice hierarchies play a crucial role in Johannson's work [24]. There is a useful converse to the existence of nice hierarchies, which can be viewed as a characterisation of them. Details will shortly appear in a paper of the authors.

THEOREM 4.1. Suppose $M$ is a compact, orientable, irreducible 3-manifold, and a 2-complex $X$ embedded in $M$ is given, consisting of embedded, compact, 


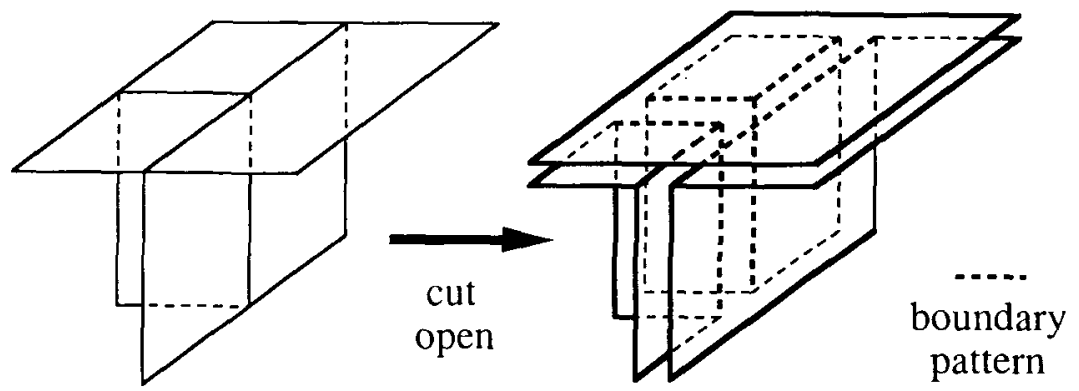

FIGURE 1

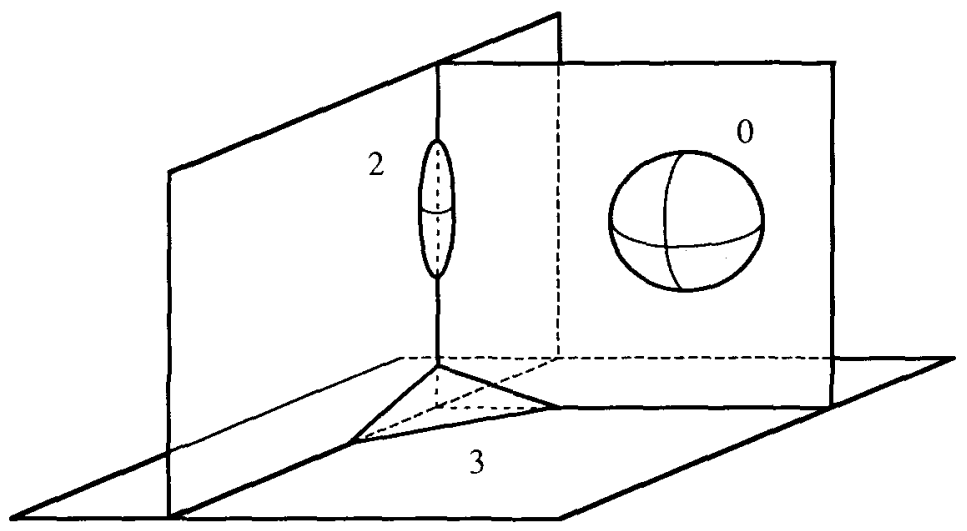

FIGURE 2. 3 choices for $D$ with $\partial D$ crossing the boundary pattern 0,2 or 3 times; $D$ parallel into the hierarchy 
orientable surfaces $S_{1}, S_{2}, \ldots S_{k}$. Assume that $S_{1}$ is closed, and that each $S_{i}$ has boundary contained in $S_{1} \cup \ldots \cup S_{i-1}$, for $2 \leq i \leq k$. Suppose there are no triangular regions in the boundary pattern of these surfaces, and that any disk $D$ embedded in $M$ with $D \cap X=\partial D$ meeting the boundary pattern in at most 3 points is parallel into $X$ (see Figure 2). Then each of the surfaces $S_{i}$ is incompressible and boundary incompressible in the manifold obtained by cutting $M$ open along $S_{1}, \ldots S_{i-1}$.

REMARK 4.1. If $M-X$ is a collection of open 3-cells, then $X$ is a hierarchy for $M$. However we can also 'fill in' the rest of $M$, by handlebodies for example, as occurs in surgery on links.

EXAMPLE 4.1. Suppose $M$ is a compact, orientable, irreducible 3-manifold satisfying either

(1) $\partial M$ consists of $n$ incompressible tori, for $n \geq 3$, or

(2) two incompressible tori, and $H_{1}(M, Z)$ has rank 2 with cup product of the generators zero, or

(3) one incompressible torus and $H_{1}(M, Z)$ has rank at least 2.

Then we can form a "non-separating" hierarchy by starting with $n-1$ boundary tori, or a closed, incompressible, non-separating surface, and subsequently choosing non-separating surfaces with non-empty boundary. The result is a "hierarchy", whose complement consists of a copy of $T^{2} \times I$. Assume that surgery is performed on the last boundary torus, with the solid torus glued on with meridian curve meeting the boundary pattern at least four times. Then adding a meridian disk of the solid torus gives a new collection of surfaces satisfying the hypotheses of Theorem 4.1. Thus the other surfaces remain incompressible, including the other boundary tori.

$\mathrm{Wu}$ has proved a closely related result [51] by completely different methods.

EXAMPLE 4.2. As a more concrete example, choose $M$ to be the complement of the Whitehead link in $S^{3}$ (see Figure 3).

Choose $S_{1}$ to be a boundary torus, $S_{2}$ a once-punctured torus (Seifert surface for $C_{1}$ ), with boundary on $S_{1}$, and $S_{3}$ a disk. The resulting boundary pattern is shown in Figure 4.

Hence only the trivial surgery on the boundary torus for $C_{2}$ makes the torus $S_{1}$ compressible, since only one essential curve on the torus in Figure 4 meets the boundary pattern less than 4 times. 


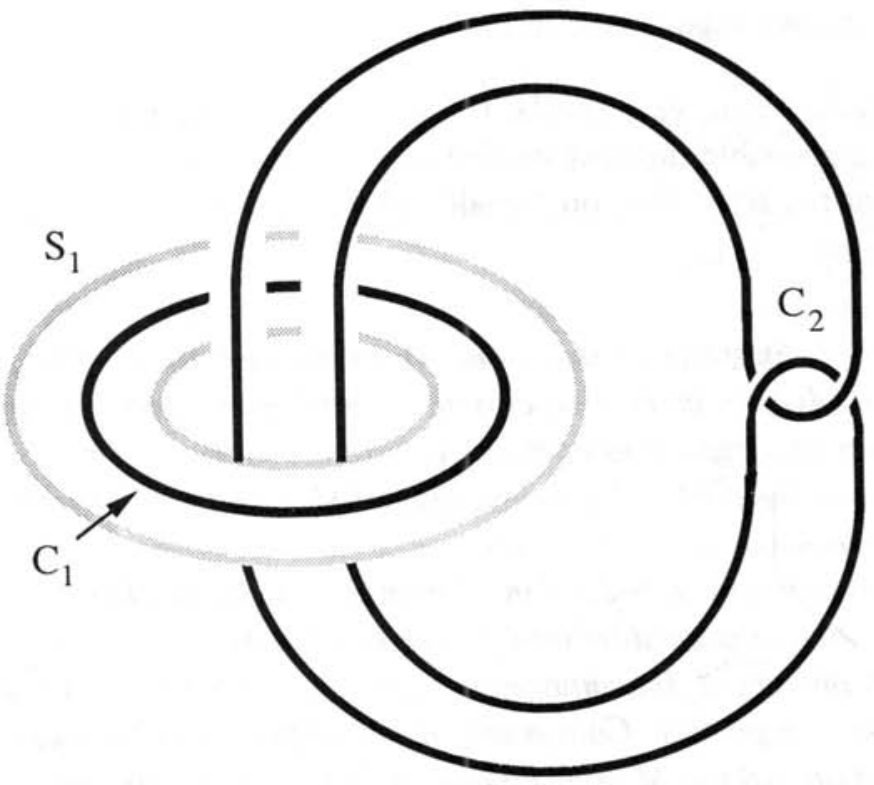

FIGURE 3

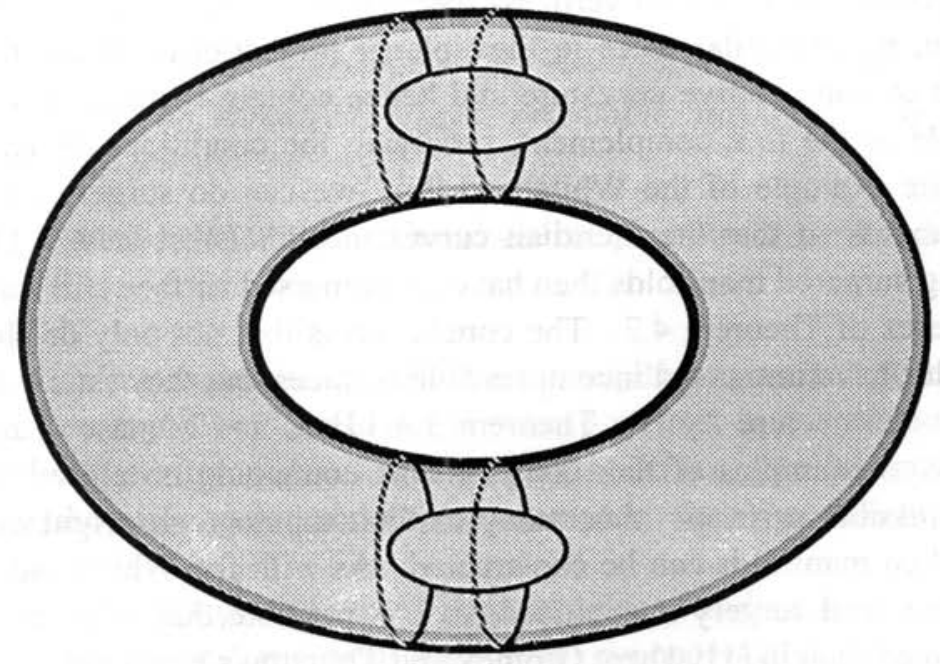

FIGURE 4 
This hierarchy also gives rise naturally to a polyhedral metric of non-positive curvature on the Whitehead link complement.

The following result, very similar to Theorem 4.1, characterises most of the singular incompressible surfaces studied by Hass and Scott [15] and the authors [3]. Note that the restriction on "small disks" is closely related to Gromov's hyperbolic groups [11].

THEOREM 4.2. Suppose $M$ is a compact, orientable, irreducible 3-manifold and $f: L \rightarrow M$ is an immersed, closed, orientable surface. Let $S(L)$ denote the double arcs and triple points of $f(L)$. Suppose that

(1) no component of $f(L)-S(L)$ is a disk with boundary containing at most 3 triple points, and

(2) if $D$ is any disk embedded in $M$ with $D \cap f(L)=\partial D$ meeting $S(L)$ at most 3 times, then $D$ is parallel into $f(L)$ as in Figure 2 (suitably modified). Then $L$ is an immersed, incompressible surface satisfying the 4-plane, 1-line and triple point properties. Conversely, if $L$ satisfies all these properties, then the conditions on disks in $M-f(L)$ and in $f(L)-S(L)$ are satisfied.

EXAMPLE 4.3. A large class of links, namely well-balanced links, are defined and studied in [1]. These are prime, non-splittable, alternating links, with exactly one 2-gon (disks with two vertices) meeting each vertex of the link diagram, and with no triangular faces in their planar projections. These have 'ideal' cubings of non-positive curvature and hence contain surfaces $f: L \rightarrow M$, where $M$ is the link complement, satisfying the conditions of Theorem 4.2. As in the example of the Whitehead link, we can do surgery on these link complements so that the meridian curves meet $S(L)$ at least 4 times. The resulting surgered manifolds then have an immersed surface still satisfying the hypotheses of Theorem 4.2. The conclusion is that not only do the surgered manifolds have immersed incompressible surfaces, but they satisfy topological rigidity: Conjecture 2.1 , by Theorem 3.3. Hass and Menasco (this volume) have given examples of link complements containing no closed, embedded, incompressible surfaces: thus many explicit topologically rigid examples of non-Haken manifolds can be constructed. As with the Whitehead link, often only the trivial surgery is excluded, so it is possible that stronger results can be obtained than in [1], where Gromov and Thurston's negatively-curved Dehn surgery was used. 
EXAMPLE 4.4. In unpublished work of the authors, a different construction of polyhedral metrics of non-positive curvature on alternating link complements has been found. We discuss this very briefly. Suppose a non-splittable, prime, alternating link is given. Then it turns out that the complement can be cubed in such a way that the boundary tori are unions of faces of the cubes. (In [1], the link is at ideal vertices of the cubes). This gives a very elementary proof that the complement has a polyhedral metric of non-positive curvature.

A planar projection of the alternating link can be 2-coloured in chessboard style, with adjacent regions coloured (say) black and white. Assume that for one colour, say black, there are no regions which are 2-gons. Then we can show there is an immersed, closed, orientable, incompressible surface in the link complement, which is totally geodesic in the polyhedral metric. However, the surface is not in general position, so only satisfies the 4-plane and triple-point conditions, but not necessarily the 1-line property. A characterisation of such surfaces can be given, similar to that in Theorem 4.2. Surgeries on the link can be described as 'good' if the meridian curves cross the double arcs of the surface at least 4 times. As almost all surgeries are good, most surgeries give closed, orientable 3-manifolds containing immersed, closed, orientable, incompressible surfaces.

The following conjecture, if positively resolved, would give good evidence for the ubiquity of immersed, incompressible surfaces.

CONJECTURE 4.1. The complement of any simple link in $S^{3}$, which is not a torus link, contains an immersed, closed, orientable, incompressible surface with no accidental parabolics.

For some results in the embedded case, see the paper by Adams and Reid in this volume.

REMARK 4.2. In unpublished work of Brunner, Neumann and Rubinstein, it is shown that any closed braid on at least 3 strings in a solid torus has an immersed, closed, orientable, incompressible surface. No information on accidental parabolics is known about these examples. They show the following.

PROPOSITION 4.1. Suppose $M$ is a compact surface bundle over a circle with fibre either a punctured torus or a three-times punctured disk. Then $M$ has a finite-sheeted cover with $H_{1}(M, Z)$ having rank larger than the number of boundary tori. 
4.1. Finding immersed incompressible surfaces. Suppose $M$ is a compact, orientable, irreducible 3-manifold and $f: L \rightarrow M$ is an immersed, closed, orientable, incompressible surface. If $\partial M \neq \emptyset$, we assume $\partial M$ is incompressible and that $L$ is not boundary parallel. By [8], after replacing $f$ by a least-area map in its homotopy class, we can suppose that $f$ lifts to an embedding $\tilde{f}: L \rightarrow M_{L}$, which induces an isomorphisim of fundamental groups. It is easy to show that the closures of the two components of $M_{L}-\tilde{f}(L)$ are non-compact, that is, $M_{L}$ has two ends. So a properly embedded line $l$ can be chosen in $M_{L}$ which runs between these two ends, meeting $\tilde{f}(L)$ transversely an odd number of times.

Conversely, suppose that $f: L \rightarrow M$ is an immersed, closed, orientable surface, not necessarily incompressible, in a compact, orientable 3-manifold with $\partial M$ incompressible. If there is a properly embedded line $l$ in $M_{L}$ which meets $\tilde{f}(L)$ transversely in an odd number of points, where $\tilde{f}: L \rightarrow M_{L}$ is a lift of $f$, then $M_{L}$ has at least 2 ends. By compressing a closed, orientable surface which separates these ends (a suitable component of the boundary of a neighbourhood of $\tilde{f}(L)$ will do), we get an embedded, closed, orientable, incompressible surface in $M_{L}$. Projecting to $M$ completes a sketch of a proof of the following result.

THEOREM 4.3. A compact, orientable, irreducible 3-manifold $M$ has an immersed, closed, orientable, incompressible surface which is not boundary parallel if and only if there is an immersed, closed, orientable surface $f: L \rightarrow M$ and a properly embedded line $l$ in $M_{L}$, with l meeting the image of some lift $\tilde{f}: L \rightarrow M_{L}$ transversely in an odd number of points.

REMARK 4.3. (1) This result can also be stated as: $M$ has an immersed, incompressible surface if and only if it has a cover with at least two ends. The viewpoint given may be useful in developing an algorithm to detect such surfaces.

(2) To make this result more usable, it would be beneficial to know that the line $l$ could be chosen to be periodic, that is, the lift of a loop $g: S^{1} \rightarrow M$. We sketch why this is true if $M$ is hyperbolic, but at present do not have a general argument.

If $M$ is hyperbolic and $L$ is incompressible and geometrically finite, then the domain of discontinuity for $L$ descends to two open sets at infinity at the two ends of $M_{L}$. It is then easy to choose a periodic geodesic line between these two open sets, as periodic geodesis are dense in the space of all geodesic lines. The periodic geodesic line $l$ will cover a geodesic loop $g: S^{1} \rightarrow M$. 
If $L$ is incompressible and geometrically infinite, then by Thurston [45], $M$ is finitely covered by an $L$-bundle over $S^{1}$. A loop in this bundle projecting onto the base $S^{1}$ clearly descends to a curve $g: S^{1} \rightarrow M$ which lifts to a line in $M_{L}$ between the two ends, as required.

(3) In Skinner [41], a solution of the word problem is given in 3-manifolds containing immersed, incompressible surfaces satisfying the 1-line and 1-point properties. The latter is that two double lines of intersection of the planes in the universal cover meet in at most one point. This is a geometric rather than a homotopy theoretic condition, but is satisfied by totally geodesic surfaces in case of metrics of non-positive curvature. Other examples are given in [41]. The main result of [41] can be interpreted as showing that for an incompressible surface of this type, there is an algorithm for deciding if an arc with ends on the surface is homotopic into the surface. So in this case, testing loops $g: S^{1} \rightarrow M$ for the required property is algorithmic.

4.2. Non-zero degree maps. A result related to Theorem 4.3 is the following.

THEOREM 4.4. Suppose $M, M^{\prime}$ are compact, orientable, irreducible 3-manifolds and $g:(M, \partial M) \rightarrow\left(M^{\prime}, \partial M^{\prime}\right)$ is a non-zero degree map. If $M^{\prime}$ has an immersed, closed, orientable, incompressible non boundary-parallel surface, then so does $M$. Moreover, if the surface in $M^{\prime}$ satisfies the 4-plane property, then so does the pull-back surface in $M$.

REMARK 4.4. (1) The idea of the proof is to use the fact that if $f: L \rightarrow M^{\prime}$ is an incompressible immersion, then $M_{L}^{\prime}$ has 2 ends. Consequently, it can be easily shown that some component of $\tilde{g}^{-1}(\tilde{f}(L))$ also separates $\tilde{M}$ with 2 ends, where $\tilde{M}$ is the covering of $M$ corresponding to the subgroup $g_{*}^{-1}\left(f_{*} \pi_{1}(L)\right)$, and $\tilde{g}: \tilde{M} \rightarrow M_{L}^{\prime}$ is a lift of $g$. Using least area techniques as in [8], it follows that the 4-plane property pulls back to $M$, after compressing the chosen component of $\tilde{g}^{-1}(\tilde{f}(L))$.

(2) An interesting special case of non-zero degree maps is branched coverings. This shows that incompressible surfaces 'lift', in a suitable sense, to branched coverings.

A 'folk theorem', known to G. Swarup and P. Scott, gives another important criteria for immersed, incompressible surfaces. The proof follows easily from Scott's core theorem (see [36] or [38]). 
THEOREM 4.5. Suppose $M$ is a compact, orientable, irreducible 3-manifold. Then $M$ has an immersed, closed, orientable, incompressible surface if and only if $\pi_{1}(M)$ has a finitely-generated subgroup which is not free and has infinite index.

\section{References}

[1] I. R. Aitchison, E. Lumsden and J. H. Rubinstein, 'Cusp structure of alternating links', Invent. Math. 109 (1992), 473-494.

[2] I. R. Aitchison and J. H. Rubinstein, 'Geodesic surfaces in knot complements', preprint, University of Melbourne, 1990.

[3] _ 'An introduction to polyhedral metrics of non-positive curvature on 3-manifolds', in: Geometry of Low-Dimensional Manifolds: 2 (eds. S. K. Donaldson and C. B. Thomas), London Math. Soc. Lecture Notes 151 (Cambridge University Press, Cambridge, 1990) pp. $127-161$.

[4] - 'Canonical surgery on alternating link diagrams', in: Knots 90 (ed. A. Kawauchi) (de Gruyter, Berlin, 1992) pp. 543-558.

[5] — 'Combinatorial cubings, cusps and the dodecahedral knots', in: Topology 90 (eds. Apanasov, Neumann, Reid and Siebenmann) (de Gruyter, Berlin, 1992) pp. 17-26.

[6] M. Culler and P. Shalen, 'Varieties of group representations and splittings of 3-manifolds', Ann. of Math. 117 (1983), 109-146.

[7] D. Epstein, 'Periodic flows on three-manifolds', Ann. of Math. 95 (1972), 66-82.

[8] M. Freedman, J. Hass and P. Scott, 'Least area incompressible surfaces in 3-manifolds', Invent. Math. 71 (1983), 609-642.

[9] D. Gabai, 'Homotopy hyperbolic 3-manifolds are virtually hyperbolic', preprint, 1992.

[10] C. Gordon and J. Luecke, 'Knots are determined by their complements', J. Amer. Math. Soc. 2 (1989), 371-415.

[11] M. Gromov, 'Hyperbolic groups', in: Essays in Group Theory (ed. S. Gersten), MSRI publications 8 (Springer, Berlin, 1987) pp. 75-264.

[12] W. Haken, 'Theorie der Normal Flächen', Acta Math. 105 (1961), 245-375.

[13] _ 'Über das Homöomorphieproblem der 3-Mannigfaltigkeiten I', Math. Z. 80 (1962), 89-120.

[14] - 'Some results on surfaces in 3-manifolds', in: Studies in Modern Topology (Math. Assoc. Amer., Washington D.C., 1968) pp. 34-98.

[15] J. Hass and P. Scott, 'Homotopy equivalence and homeomorphism of 3-manifolds', Topo$\log y 31$ (1992), 493-517.

[16] A. Hatcher, 'On the boundary curves of incompressible surfaces', Pacific J. Math. 99 (1982), 373-377.

[17] G. Hemion, 'On the classification of homeomorphisms of 2-manifolds and the classification of 3-manifolds', Acta Math. 142 (1979), 123-155.

[18] J. Hempel, 3-Manifolds, Annals of Math. Studies 86 (Princeton University Press, Princeton, 1976).

[19] — 'Homology of coverings', Pacific J. Math. 112 (1984), 83-113. 
[20] _ - 'Residual finiteness for 3-manifolds', in: Combinatorial group theory and topology, volume 111 of Annals of Math. Studies (Princeton University Press, Princeton, 1987) pp. 379-396.

[21] W. Jaco, Lectures on three-manifold topology, Conf. Board of Math. Sci. 43 (American Math. Society, Providence, 1980).

[22] W. Jaco and J. H. Rubinstein, 'PL minimal surfaces in 3-manifolds', J. Differential Geom. 27 (1988), 493-524.

[23] W. Jaco and P. Shalen, Seifert Fibered Spaces in 3-Manifolds, Mem. Amer. Math. Soc. 220 (1980).

[24] K. Johannson, Homotopy Equivalences of 3-manifolds with Boundary, Lecture Notes in Mathematics 761 (Springer-Verlag, Berlin, 1979).

[25] H. Kneser, 'Geschlossene Flächen in dreidimensionalen Mannigfaltigkeiten', Jahresber. Deutsch Math.-Verein 38 (1929), 248-260.

[26] W. B. R. Lickorish, 'A representation of orientable combinatorial 3-manifolds', Ann. of Math. 76 (1962), 531-540.

[27] D. Long, 'Immersions and embeddings of totally geodesic surfaces', Bull. London Math. Soc. 19 (1987), 481-484.

[28] A. A. Markov, 'Unsolvability of the problem of homeomorphy', in: Proceedings of the International Congress of Mathematicians 1958 (ed. J. A. Todd) (Cambridge University Press, Cambridge, 1960) pp. 300-306.

[29] J. Millson, 'On the first Betti number of a constant negatively curved manifold', Ann. of Math. 104 (1976), 235-247.

[30] J. Milnor, 'A unique factorisation theorem for 3-manifolds', Amer. J. Math. 84 (1962), 1-7.

[31] E. Moise, 'Affine structures in 3-manifolds V. the triangulation theorem and hauptvermutung', Ann. of Math. 56 (1952), 96-114.

[32] G. Mostow, 'Quasi-conformal mappings in n-space and the rigidity of hyperbolic space forms', Inst. Hautes Études Sci. Publ. Math. 34 (1968), 53-104.

[33] P. Orlik, Seifert Manifolds, Lecture Notes in Mathematics 291 (Springer-Verlag, Berlin, 1972).

[34] C. Papakyriakopoulos, 'On Dehn's Lemma and the asphericity of knots', Ann. of Math. 66 (1957), 1-26.

[35] _ ' 'On solid tori', Proc. London Math. Soc. 7 (1957), 281-299.

[36] J. H. Rubinstein and G. A. Swarup, 'On Scott's core theorem', Bull. London Math. Soc. 22 (1990), 495-498.

[37] H. Schubert, 'Bestimmung der Primfaktorzerlegung von Verkettungen', Math. Zeit. 76 (1961), 116-148.

[38] P. Scott, 'Compact submanifolds of 3-manifolds', J. London Math. Soc. (2) 7 (1973), 246-250.

[39] _ 'The geometries of 3-manifolds', Bull. London Math. Soc. 15 (1983), 401-487.

[40] H. Seifert, 'Topologie dreidimensionalen gefaserter Räume', Acta Math. 60 (1933), 147238.

[41] A. Skinner, The word problem in the fundamental groups of a class of three dimensional manifolds (Ph.D. Thesis, University of Melbourne, 1991).

[42] J. Stallings, 'On the loop theorem', Ann. of Math. 72 (1960), 12-19.

[43] —, 'On fibering certain 3-manifolds', in: Topology of 3-Manifolds (ed. M. Fort) (Prentice-Hall, 1962) pp. 95-100.

[44] G. A. Swarup, 'On a theorem of Johannson', J. London Math. Soc. 18 (1978), 560-562. 
[45] W. Thurston, The geometry and topology of 3-manifolds (Lecture notes, Princeton University, 1978).

[46] - 'Three dimensional manifolds, Kleinian groups and hyperbolic geometry', Bull. Amer. Math. Soc. 6 (1982), 357-381.

[47] _- 'Hyperbolic structures on 3-manifolds I: Deformations of acylindrical manifolds', Ann. of Math. 124 (1986), 203-246.

[48] F. Waldhausen, 'On irreducible 3-manifolds which are sufficiently large', Ann. of Math. 87 (1968), 56-88.

[49] _ 'The word problem in fundamental groups of sufficiently large 3-manifolds', Ann. of Math. 88 (1968), 272-280.

[50] $\longrightarrow$, On the determination of some bounded 3-manifolds by their fundamental groups alone', in: Proc. of Inter. Sym. on Topology (Yugoslavia, Beograd, 1969) pp. 331-332.

[51] Y-Q. Wu, 'Incompressibility of surfaces in surgered 3-manifolds', Topology 31 (1992), 271-279.

Mathematics Department

University of Melbourne

Parkville, Victoria

Australia 3052
Mathematics Department

University of Melbourne

Parkville, Victoria

Australia 3052 